\title{
Modified Image Thresholding using Social Impact Theory based Optimization (SITO)
}

\author{
Harpreet Kaur \\ ME-CSE \\ CU, Gharuan \\ Mohali, Punjab, India
}

\author{
Shruti Mittal \\ Assistant Professor-CSE \\ CU, Gharuan \\ Mohali, Punjab, India
}

\begin{abstract}
Thresholding is considered as pivotal tool for image segmentation [1]. The main aim of thresholding is to divide the pixels into different groups in a logical way [2]. One of the most suitable algorithm for thresholding is Social Impact Theory Based Optimization (SITO).Social Impact theory optimization algorithm has been considered as one of the important technique to find the better optimized results as it is based on human behavior. The cross entropy function works well in case of bi-level thresholding problem. However, if there is a need of the multi-thresholding in image processing application, a global and generic objective function is desired so that each threshold could be tested for its best performance statistically [6]. The maxima of the selected threshold are optimized by using the SITO algorithm based on maxima of sum of entropy ad standard deviation. The results are compared with negative selection algorithm (NSA) which is artificial intelligence (AI) technique, maximum entropy algorithm and OTSU algorithm. The performance measures i.e. Standard Deviation, Entropy, MSE and PSNR prove the improvements of SITO based thresholding.
\end{abstract}

\section{Keywords}

SITO, Thresholding, NSA, MSE, PSNR

\section{INTRODUCTION}

Image Thresholding is defined as the process of separating an image into different groups. Each group contains number of pixels known as super pixels. Image segmentation is a method, which is used for locating the objects and boundaries (lines, curves, etc.) in different images. The main aim of Image segmentation is basically to distribute or group an image into distinct parts known as regions. Image thresholding is the procedure of allotting a name to each pixel in the given image [4] .After thresholding the result comes out is the set of different segments covering the whole image. The thresholding is basically based upon measurements that are taken from an image. The factors on which thresholding depends are basically grey level of an image, texture of an image, depth of an image, color of an image or motion of an image. As image thresholding is of great importance therefore based on the given images different number of algorithms has been acknowledged [13] .These algorithms show best thresholding results at the end.

The image thresholding algorithm used for monochrome images mainly used for the property of intensity values of pixels based on the discontinuity and similarity. The most widely used color driven thresholding techniques include clustering, histogram based thresholding, edge detection, region growing, neural networks thresholding, compression based, watershed transformation, multi-scale segmentation, graph partitioning methods [7]. These techniques have the aim to reduce the number of color components from the input image. The main characteristics used as the basis of color image thresholding are color and texture [5]. Color histograms are basically used in content-based healing systems and have demonstrated to be convenient.

\section{PROPOSED METHODOLOGY}

\subsection{Social Impact Theory Based Optimization (SITO)}

Social Impact Theory based Optimization is a meta-heuristic algorithm which is based on the population which will imitate the behavior of human, feelings of human and thoughts of human to get the optimized solution. This algorithm was come into existence in 2006.Each individual has different feelings, beliefs, thoughts, motives, cognition and emotions [3]. SITO algorithm will work according to all these factors. Three factors are under consideration:

1. Strength: It simply denotes the importance of individuals in fluency.

2. Immediacy: It represents closeness of one individual with the other individual.

3. Number: It simply describes the number of the individuals which are influencing the actual individuals.

Firstly the set of the individuals is considered in the form of matrix. Each individual has different opinion. Taking one individual from the matrix first of all its neighbors are checked with respect to it .The individuals with opposing beliefs are known as persuaders whereas the individuals with the same belief are known as supporters [8]. After matching the whole society of individuals will converge to one single and best optimal solution. Different number of iterations is performed in the same way. By this way we get optimized results.

\subsection{Extraction of Thresholded Value}

Best threshold value $=($ Sum of entropies + Sum of Standard deviation)

The proposed algorithm is implemented on different inbuilt MATLAB images.

\subsection{Fitness Function}

In the applied technique, Fitness function is computed that basically means to detect the best threshold value from the image [9]. To obtain the fitness function for image thresholding using SITO algorithm, the entropy and standard deviation of the given image is calculated at each pixel. Then the histogram is drawn. At point where the entropy and 
thresholding histogram meet is considered as the best thresholding point with best thresholding value.

Fitness Function $\mathrm{F}(\mathrm{x})$ is given by:

$$
\begin{gathered}
\mathrm{F}(\mathrm{x})=\operatorname{maxima}(\text { Entropy }+\mathrm{SD}) \\
F(x)=\max \left[-\sum_{i=0}^{L-1} p_{i \ln p_{i}}+\frac{1}{n \times n} \sum_{j=1}^{n} \sum_{i=1}^{m}\left(x_{i . j}-\mu\right)^{2}\right]
\end{gathered}
$$

\subsection{Proposed Algorithm}

The presented work consists of following steps:

$>$ Image Acquisition (Data Base Images)

$>$ Images are taken by using mobile camera and internet sources for testing purposes. Normally the image format taken for testing is jpeg format. Although other formats like PNG, TIFF,JPEG and BMP may also be taken for testing purposes.

$>$ RGB to Gray scale conversion

$>$ RGB to gray scale conversion is carried out by using the rgb2graycommand in matlab [14].

$>$ Computation of gray image histogram

$>$ A histogram is a graph between pixel intensities and no. of pixels of the respective pixel intensity. This is done by using the imhist command in matlab.

$>$ Initialization of loop from gray level intensity from $\mathrm{L}=0$ to 255

$>$ Computation of entropies of back ground and foreground taking threshold $\mathrm{T}=\mathrm{L}$

$$
\text { Entropy = entropy (I); }
$$

$>\quad$ Where I is the gray scale image.

$>$ Computation of standard deviation of gray level intensity taking threshold $\mathrm{T}=\mathrm{L}$

$$
\text { Std. Dev. = std (I); }
$$

$>$ Where, I is the gray scale image.

$>$ Computation of sum of Entropies and Standard Deviation

$>$ Repeat the steps from 5 to 7 . Store the sum of entropies and standard deviation in an array.

$>$ Sort the sum array for maximum of the sum.

$>$ Take the maximum sum index as threshold value.

$>$ Binarize the input image using the threshold value.

> Compute PSNR and MSE.

$>$ Binarize the given input image using Otsu and Negative Selection algorithm as well.

$>$ Compute PSNR and MSE for binary image as obtained using Otsu and negative selection algorithm.

$>$ Compare the results.

\section{RESULTS}

The performance of the proposed technique is evaluated and compared with other thresholding techniques like Otsu [16], NSA [15]. In the proposed algorithm an RGB image [13] is taken .Then it is converted into gray scale image. The results have been tabulated for four standard MATLAB images [12] For the comparison purpose Peak Signal to Noise Ratio and Mean Square Error are calculated.

PSNR: The peak-signal to noise ratio (PSNR) will tell the quality of the image which is obtained after applying the thresholded algorithm [10]. The equation is given as:

$$
\text { PSNR }=10 \log _{10} \frac{255^{2}}{\frac{1}{N \times N} \sum_{i=0}^{N-1} \sum_{\sum j=0}^{N-1}(f(i, j)-f(i, j))^{2}} d B
$$

$\mathrm{N}$ is the size of the image and $f(i, j)$ are the pixel values of an image.

MSE: Mean Square Error is given by [11]:

MSE $=\Sigma$ Gray $(\mathrm{I}, \mathrm{j})-\operatorname{SITO}(\mathrm{I}, \mathrm{j}) 2 /($ Row x Col $)$

Where Gray $(i, j)$ is the intensity of original image and SITO $(i, j)$ is the intensity of thresholded image.

Following two figures show the results obtained

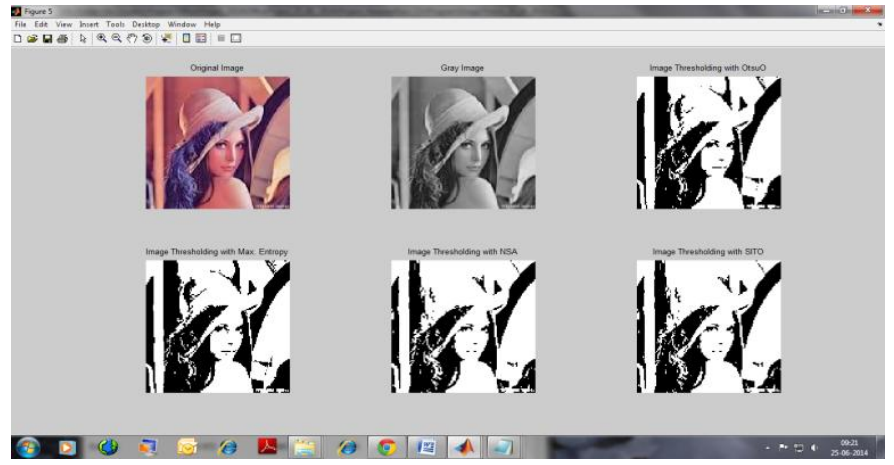

\begin{tabular}{|c|c|c|c|c|}
\hline \multicolumn{5}{|c|}{ Comparison of Image Thresholding using various methods } \\
\hline & Otsu & Max. Entropy & NSA & SITO \\
\hline Threshold & 0.412 & 0.563 & 0.412 & 0.371 \\
\hline Entropy & 0.828 & 0.504 & 0.900 & 0.900 \\
\hline MSE & 0.412 & 0.149 & 0.099 & 0.056 \\
\hline PSNR & 0.000 & 129.91 & 134. & 140 \\
\hline
\end{tabular}

Figure 1 Thresholded image using SITO algorithm

Table 1 Comparison of various techniques

\begin{tabular}{llllr}
\multicolumn{4}{c}{ Comparison of Image Thresholding using various methods } \\
& Otsu & Max. Entropy & NSA & SITO \\
& & & & \\
Threshold & 0.431 & 0.441 & 0.431 & 0.388 \\
Entropy & 0.972 & 0.980 & 0.935 & 0.935 \\
MSE & 0.431 & 0.051 & 0.119 & 0.015 \\
PSNR & 0.000 & 141 & 132.2 & 152.576
\end{tabular}

Table 2 Comparison of various techniques 


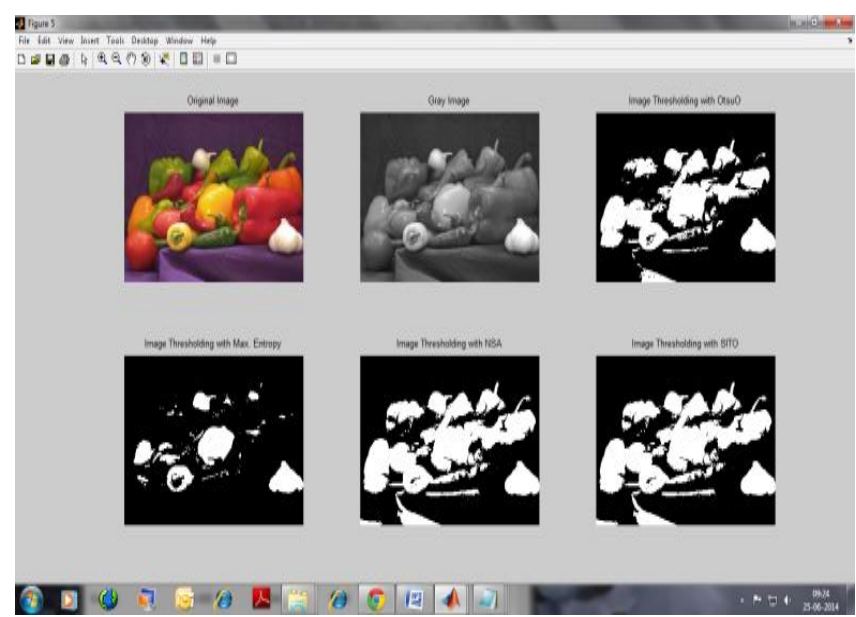

Figure 2 Thresholding using SITO algorithm

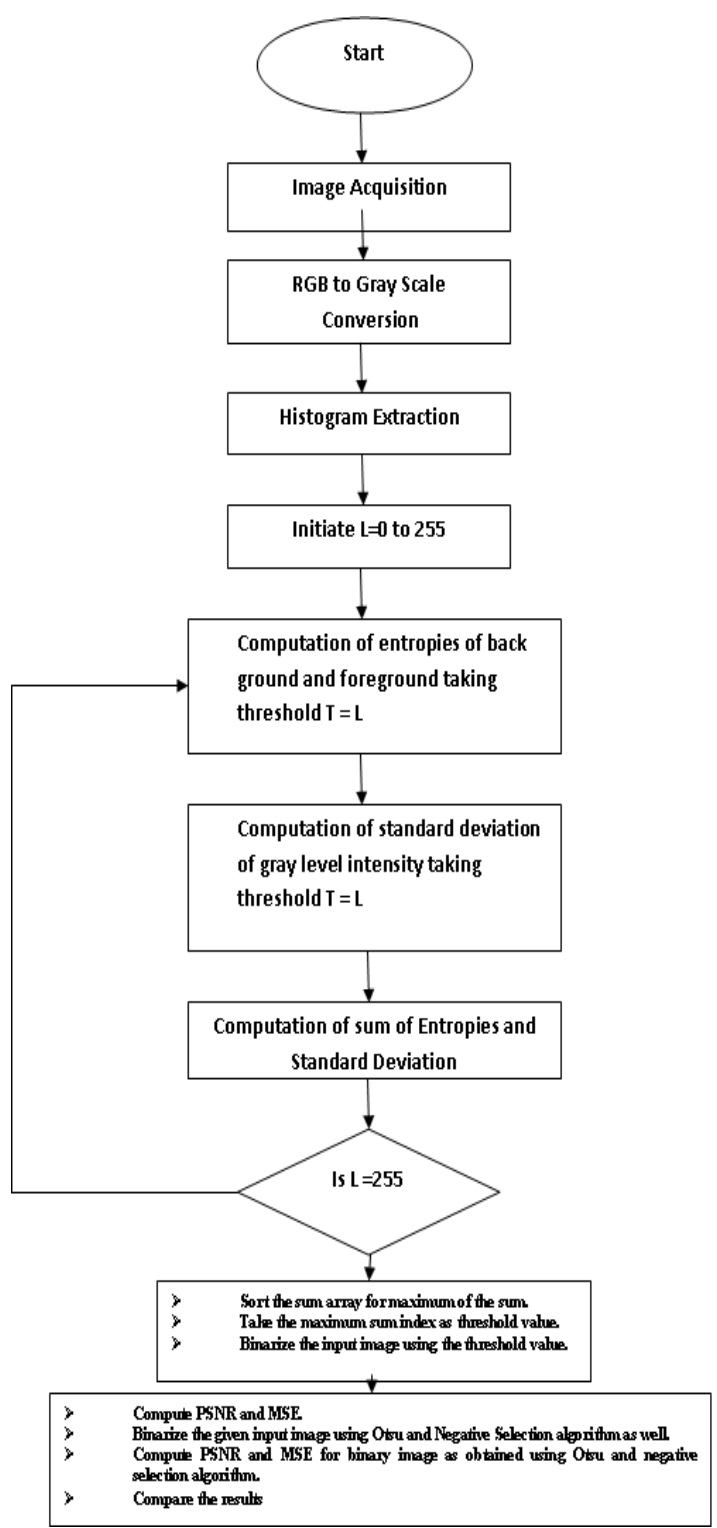

Fig.3 Proposed Algorithm

\section{CONCLUSION}

The main issue which is considered in image segmentation is how effective the thresholding is done. By comparing the results of proposed method with the existing one, the results are very optimal and satisfactory. The three important performance measures which are used in this algorithm for comparing results are the Peak to Signal Noise Ratio (PSNR), entropy and standard deviation measure and Mean Square Error. We all know that the optimization algorithms are stochastic and random searching; the results will not be same during different iterations of the algorithm. As the SITO algorithm is based on human behavior the results are very optimal and better.

\section{FUTURE SCOPE}

The proposed thesis work is based on thresholding of images using social impact theory based optimization. The images obtained after thresholding using the SITO algorithm has shown a fair improvement over the existing algorithm and can be observed from the results as obtained and discussed in results section of the report. The algorithm has scope of future work on line of improving the time required to complete the performance. As the algorithm is iterative in nature due to multiple combinations of threshold selection based on fitness function, therefore, for larger images, the time may be more. Therefore, the time dimension needs to be taken care of in future designing of the algorithm.

\section{REFERENCES}

[1] Prasant Kumar Mahapatra,Mandeep Kaur,Spardha Sethi,Rishabh Thareja,Amod Kumar,Swapna Devi,'Improved thresholding based on negative selection algorithm(NSA),"-Springer 2013.

[2] Yi Hong and Hanli Wang, "Image thresholding based on Random spatial sampling and Majority voting," Dept. of Comput. Sci.,Vol. 2, IEEE, 2010.

[3] M Macas, "Social Impact Theory Based Optimization," IEEE Transactions on Soft Computing, Vol. 23, 2001

[4] Ying Zhuge,Jayaram K.Udupa,Punam K.Saha, "Vectorial scale-based fuzzy-connected image segmentation,"Medical Image Processing Grroup,USA 2006.

[5] Guang Yang, "Study on Statistics Iterative Thresholding Segmentation Based on Aviation Image," IEEE, Vol. 2, 2007.

[6] Kamal Hammouche,Moussa Diaf,Patrick Siarry, "A multilevel automatic thresholding method based on a genetic algorithm for a fast image segmentation,"Science Direct, 2007.

[7] Kezong Tanga,Xiaojing Yuan,Tingkai Sun ,Jingyu Yang,Shang Gao, "An improved scheme for minimum cross entropy threshold selection based on genetic algorithm," Science Direct, 2011.

[8] Chang,WangJ.,Guo, "Survey and comparative analysis of entropy and relative entropy thresholding techniques," Vision,Image and Signal Processing,IEE Proceedings, Vol. 153, 2006.

[9] Minh Luan Nguyen, "Rice leaf detection with genetic programming," Computer and Information Technology, 2008 
[10] Soham Sarkar,Gyana Ranjan Patra, and Swagtam Das, "A differential Evolution Based Approach for Multilevel Image Segmentation Using Minimum Cross Entropy Thresholding,” Springer Berlin Heidelberg, 2011.

[11] Azami, H., "Segmentation of medical images based on hierarchical evolutionary and bee algorithms," IEEE, Vol. 93, 2013.

[12] Suhre,A.;Kose,K;Cetin,A.E., "Image Compression using a histogram-based color transform," IEEE Trans. Evol. Comput., 9(1), pp. 61-73, 2010.

[13] M. Tripathy, and S. Mishra, "Bacterial foraging based solution to optimize both real power and voltage stability limit," IEEE Trans.Power Syst., 22(1), pp. 240248, 2007.
[14] W. Lin, and P.X. Liu, "Hammerstein model identification based on bacterial foraging," IEE Electronics Letters, 42(23), pp. 1332-1334, 2006.

[15] P. K. Sahoo, S. Soltani, and A. K. C. Wong, "A survey of thresholding techniques,"Computer Vision, Graphics and Image Processing, vol. 41(2), pp. 233-260, 1988.

[16] C.A. Glasbey, "An analysis of histogram based thresholding algorithms," CVGIP: Graphical Models and Image Processing, Vol. 55, pp.532-537, 1993.

\section{AUTHORS PROFILE}

The author 1 is pursuing her M.E. (CSE) thesis work in Image Thresholding from CU, Gharuan, and Mohali (Punjab) India. Her field of interest is in image processing based application development 